\title{
Síndrome de Mirizzi.
}

\section{Reporte de cuatiro casos}

Laguna-Teniente $\mathbb{I R}^{*}$, Flores-Alvarez $E^{* *}$

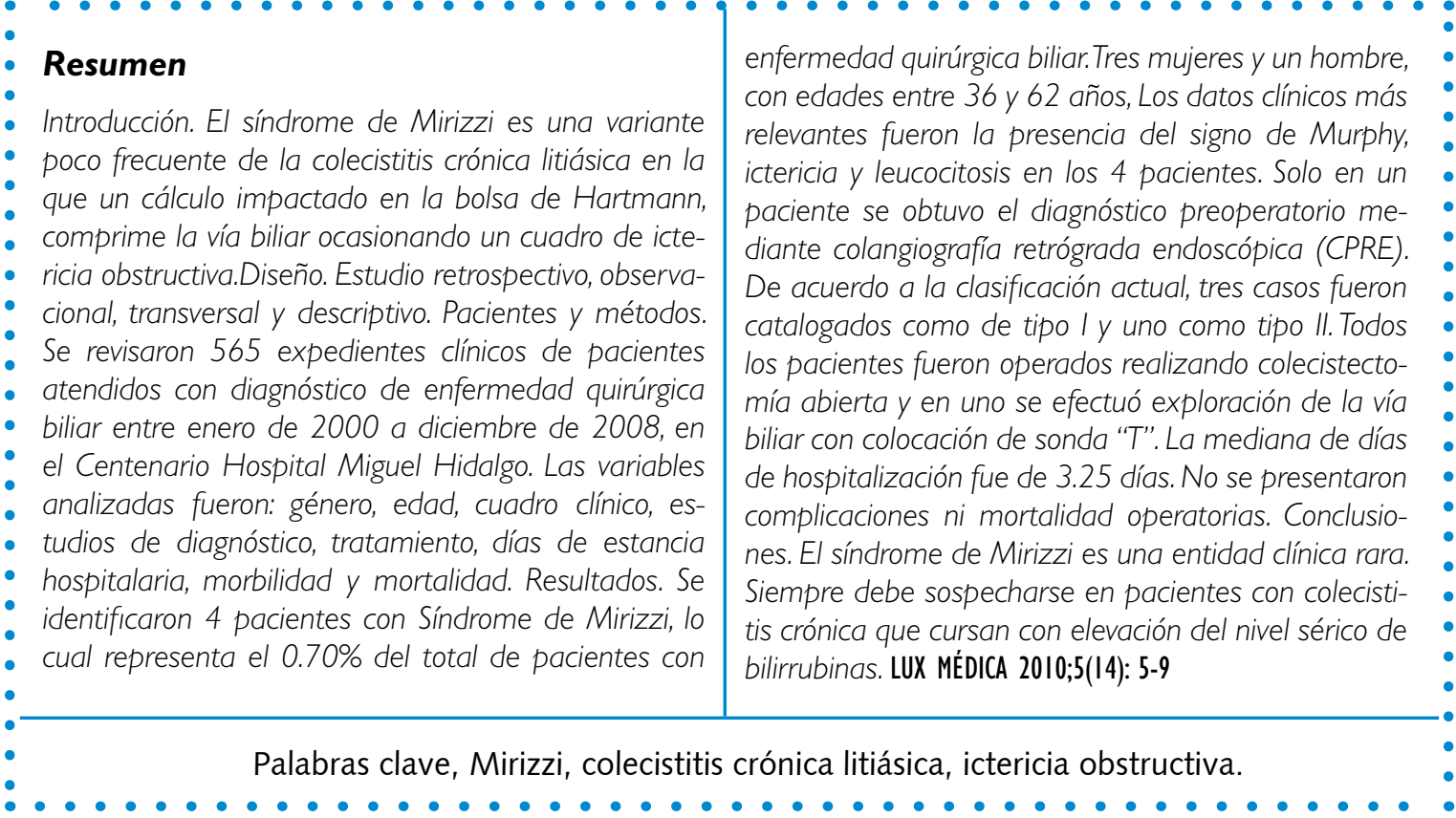

\section{Introducción}

El síndrome de Mirizzi es una entidad clínica infrecuente reportada por primera vez en 1948, por Pablo Luís Mirizzi. Descrita como un cuadro de ictericia obstructiva ocasionada por la compresión del conducto hepático común por un cálculo en el conducto cístico o en el cuello vesicular ${ }^{1}$. La secuencia etiopatogénica inicia con una colelitiasis, seguida por el impacto de un lito sobre la bolsa de Hartmann o el conducto cístico, finalizando en una colestasis por compresión extrínseca de la vía biliar principal². Pueden desencadenarse eventos como ictericia clínica o subclínica, exclusión biliar,

* Médico residente de Cirugía General, Centenario Hospital Miguel Hidalgo Ags.

** Cirujano General. Cirujano Oncólogo. Maestro en Ciencias. Profesor titular del curso de Cirujía General. Centenario Hospital Miguel Hidalgo Ags.. 
colecistitis aguda, fístula colecisto-coledociana, colangitis y cirrosis biliar. Generalmente se comporta como un cuadro silente y puede encontrarse como hallazgo en el transcurso de una colecistectomía, por lo que la mayoría de los casos se diagnostica durante un evento quirúrgico ${ }^{3}$. El objetivo del estudio es reportar cuatro casos de síndrome de Mirizzi tratados en nuestro hospital.

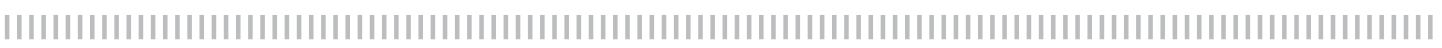

\section{Pacientes y métodos}

Se realizó un estudio retrospectivo, observacional, transversal y descriptivo en el servicio de Cirugía General del Centenario Hospital Miguel Hidalgo, en un periodo comprendido entre enero de 2000 y diciembre de 2008. De un total 565 expedientes clínicos de pacientes con enfermedad biliar quirúrgica, se analizaron cuatro pacientes con diagnóstico confirmado de síndrome de Mirizzi. Las variables estudia- das fueron: presencia de signo de Murphy, ictericia, leucocitosis, hallazgos en ultrasonido, colangiopancreatografía retrógrada endoscópica (CPRE), tomografía axial computarizada (TAC), tipo de Mirizzi (IIV), tipo de colecistectomía, exploración de vía biliar, uso de drenaje de la cavidad peritoneal, uso de sonda " $\mathrm{T}$ ", tiempo quirúrgico, complicaciones postoperatorias, días de estancia hospitalaria, evolución de los pacientes y mortalidad.

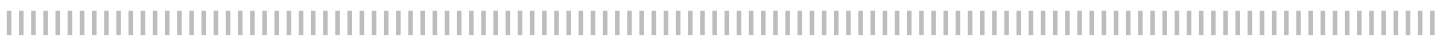

\section{Resultados}

Presentamos cuatro casos de pacientes con diagnóstico de síndrome de Mirizzi, tres mujeres y un hombre. Tres pacientes con la variante tipo I y uno con la tipo II. Un paciente fue diagnosticado preoperatoriamente por medio de CPRE, los otros tres fueron diagnosticados durante la cirugía. Todos los pacientes presentaron ictericia y signos clínicos de colecistitis aguda. Los cuatro pacientes presentaban enfermeda- des concomitantes tales como hipertensión arterial sistémica, diabetes mellitus tipo 2 y obesidad. En los cuatro pacientes se observó leucocitosis por arriba de 10,000/mm3. Los cuatro pacientes fueron sometidos a tratamiento quirúrgico efectuándose colecistectomía abierta y en uno de ellos se exploró la vía biliar y se colocó una sonda " $T$ ". En ninguno se realizó reintervención y no ocurrió ninguna defunción. Los resultados individuales se reportan en la tabla 1.

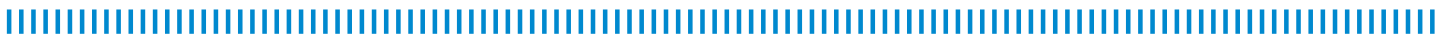

\section{Discusión}

El síndrome de Mirizzi es una entidad poco común dentro de la patología biliar, se reporta una incidencia entre 0.7 a $1.4 \%$ en los pacientes operados de colecistectomía por colecistitis crónica litiásica ${ }^{4}$. En nuestro análisis la tasa fue de $0.70 \%$. Para el desarrollo del síndrome de Mirizzi se describen cuatro componentes involucrados: (1) Paralelismo del conducto cístico o cue- llo vesicular al conducto hepático común; (2) La presencia de un lito impactado en el conducto cístico o el cuello vesicular; (3) La obstrucción del conducto hepático, e; (4) Ictericia y/o hiperbilirrubinemia. Inicialmente ocurren episodios de inflamación que provocan adherencias entre el cuello vesicular y el conducto hepático que terminan por fusionarse. Si el proceso continúa, por medio de un mecanismo de presión, el 
cálculo puede erosionar la pared e introducirse de forma parcial o total en el conducto hepatocolédoco ${ }^{5}$.

La mayor parte de los casos son diagnosticados durante la cirugía que presenta serias dificultades técnicas y constituyen un verdadero reto para el cirujano. Hoy en día, con la utilización de nuevas técnicas diagnósticas, han permitido diagnosticar a los pacientes en la fase preoperatoria. El empleo de la colangio-resonancia magnética, la tomografía axial computada helicoidal con colangiograma y la colangiopancreatografía retrógrada endoscópica permiten su detección y un plan operatorio establecido con anticipación ${ }^{6,7}$.

No existe un cuadro clínico consistente y suele ser idéntico con otras formas de obstrucción biliar. Los síntomas recurrentes, náusea, dolor en cuadrante superior derecho o alteraciones en las pruebas de bioquímica sérica, son inconstantes. La presentación aguda puede incluir pancreatitis o colecistitis ${ }^{2}$. El ultrasonido puede confirmar la naturaleza litiásica y en ocasiones distinguirla de una etiología neoplásica. La CPRE constituye el estudio más adecuado para mostrar la causa de la ictericia y permitir en algunos casos la extracción de un cálculo, alojado en una comunicación cístico-coledociana ${ }^{8}$. A todos nuestros pacientes se les realizó ultrasonido, evidenciando datos de dilatación de vía biliar, la presencia de imágenes sugestivas de cálculos y cambios anatómicos inespecíficos del cístico o vesícula biliar. Se realizó CPRE en un paciente, el cual confirmó el diagnóstico y fue operado.
La intervención quirúrgica es tanto más compleja cuando mayor sea el grado de lesión, por lo que un diagnóstico preoperatorio es fundamental para diseñar la estrategia a emplear. Algunos autores proponen como metodología ideal la colangiografía asociada a la resonancia magnética nuclear. La colangiografía intraoperatoria es útil como complemento, luego de colangiografías preoperatorias, para confirmar el diagnóstico y obtener un mapa de las vías biliares; se considera indispensable cuando no se han realizado estudios preoperato$\operatorname{rios}^{3,9}$.

La alteración de la anatomía de las vías biliares en el Síndrome de Mirizzi predispone a la lesión ductal. El procedimiento quirúrgico debe de elegirse según el tipo de lesión, pero en general es la colecistectomía, drenaje biliar, plastías o anastomosis bilio-digestiva. Existe un consenso que propone, para resolver lesiones de grado I, la colecistectomía, ya sea parcial o total; en la tipo II, la reparación del defecto y drenaje con sonda " $T$ "; en el tipo III, reparación de la pared de la vía biliar mediante un colgajo de la pared vesicular, y; en lesión tipo IV, está indicada la anastomosis biliodigestiva con una asa de yeyuno en $Y$ de Roux ${ }^{10,11}$.

En la era de la cirugía laparoscópica, este tipo de lesiones también son resueltas por esta vía, salvo en los casos muy avanzados o complicados que deben convertirse por razones obvias ${ }^{12}$. En nuestra serie, todos los casos fueron tratados por la vía convencional.

\section{Conclusiones}

El síndrome de Mirizzi es una entidad clínica rara. Siempre debe sospecharse en pacientes con colecistitis crónica que cursen con elevación del nivel sérico de bilirrubinas. 


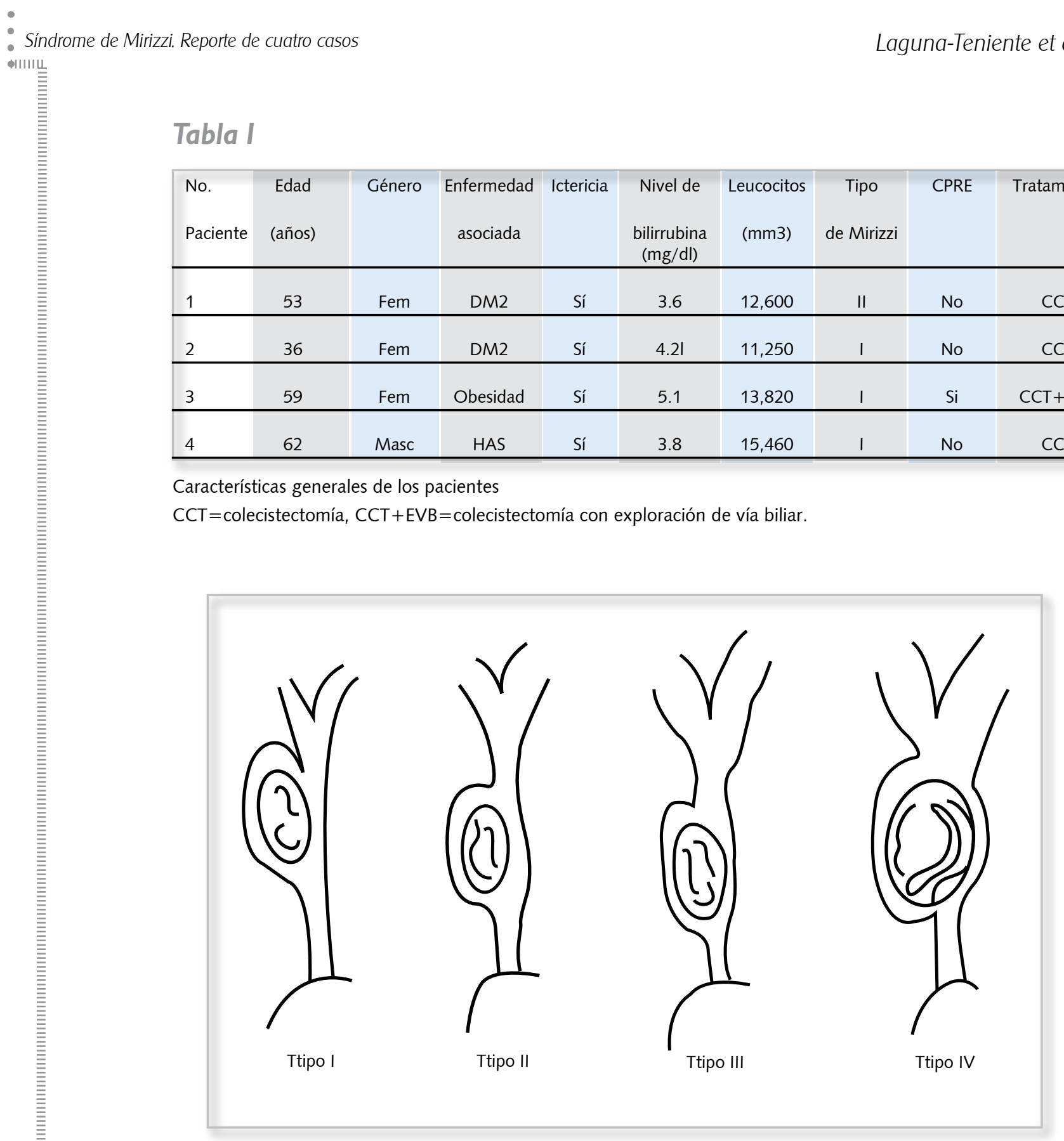

Fig 1. Clasificación de Síndrome de Mirizzi.

AGRADECIMIENTO A Laura Lorena Flores Tavizón por la realización de las tablas y figuras. 


\section{Tabla 2}

\section{Características del Síndrome de Mirizzi}

\begin{tabular}{|l|l|l|}
\hline Tipo & Características & Tratamiento \\
\hline I & $\begin{array}{l}\text { Compresión externa del conducto biliar común debido a un calculo impactado } \\
\text { en el cuello de la vesícula biliar o en conducto cístico, sin fístula. }\end{array}$ & Colecistectomía \\
\hline II & $\begin{array}{l}\text { Fístula colecistobiliar, involucra menos del 33\% de la circunferencia del } \\
\text { conducto biliar. }\end{array}$ & Colecistectomía \\
\hline III & Fístula con erosión del $33-66 \%$ de de la circunferencia del conducto biliar. & $\begin{array}{c}\text { Colecistectomía } \\
+ \text { Plastia del } \\
\text { conducto biliar }\end{array}$ \\
\hline IV & Fístula que involucro el $66-100 \%$ del diámetro del conducto biliar. & $\begin{array}{l}\text { Colecistectomía } \\
+ \text { Plastía del } \\
\text { conducto biliar }\end{array}$ \\
\hline
\end{tabular}

\section{Bibliografía}

1 Juárez $C D$, Hurtado DJL, Flores GS, Sentíes-Carvajal SB. Síndrome de Mirizzi: experiencia en siete pacientes. Cir. Gen 2001; 23: 99-104.

2 Csendes A, Díaz JC, Burdiles P, Maluenda F, Nava O. Mirizzi syndrome and cholecystobiliary fistula: a unifying classification. Br J Surg 1989; 76:1139-43.

3 Tan KY, Chung HC, Chen CY, Tan SM, Poh BK, Hoe MN. Mirizzi syndrome: noteworthy aspects of a retrospective study in one centre. ANZ J Surg 2004; 74:833-7.

4 Espino $\mathrm{CH}$, Bernal SF, Murguía DD, Valdés LR. Síndrome de Mirizzi: una causa rara de ictericia obstructiva. Rev Gastroenterol Méx 1993; 58: 25-30.

5 Tanaka N, Nobori M, Furuya T, Ueno T, Kimura H. Evolution of Mirizzi syndrome with biliobiliary fistula. J Gastroenterol 1995; 30: 117-121.

6 Castor-Samaniego A, Valeria-Sanabria Z, Aníbal-Filártiga I. Experiencia en tratamiento del Síndrome de Mirizzi. Rev Chilena Cir 2006;58(4):276-280.

7 Tulassay Z. Endoscopic retrograde cholangiopancreatography in Mirizzi syndrome. Am J Gastroenterol 1987; 82: 391-392.
8 Castellón CJ, Fernández M, Del Amo E. Coledocolitiasis: indicaciones de colangiopancreatografía retrógrada endoscópica y colangiorresonancia magnética. Cir Esp 2002; 71: 314-8.

9 Sando N, Saito $H$. The efficacy of pre and postoperation magnetic resonance cholangiopancreatography for biliary tract disease. Hepatogastroenterol 1998; Supp II, Vol 45. Abstracts 3rd World Congress.

10 Krahenbuhl L, MoserJ, Redaelli C, Seiler Ch, Maurer Ch, Baer H. A Standardized Surgical Approach for the Treatment of Mirizzi Syndrome. Dig Surg 1997; 14: 272-276.

11 Bare HU, Matthews JB, Schweizer WP, Gertsch P, Blumgart LH. Management of the Mirizzi syndrome and the surgical implications of cholecistocholedochal fistula. Br J Surg 1990; 77: 743-745.

12 Robles Palomar PJ, Lancaster Jones B, García Lara J. Síndrome de Mirizzi, abordaje abdominal laparoscópico. Rev Mex Cir Endoscop 2005; 6: 55-8. 